\title{
Correction to: An argument against global no miracles arguments
}

\author{
Florian J. Boge $\mathrm{e}^{1,2}$
}

Published online: 2 March 2020

(c) Springer Nature B.V. 2020

\section{Correction to: Synthese https://doi.org/10.1007/s11229-018-01925-9}

- The sentence

"The success of the older theories will not entail any genuine success of a new one, in the sense specified above, and one could hence reasonably maintain that older successes have no say in the estimation of the new theory's success."

in the final paragraph of section 2.1 needs to be

"The success of the older theories will not be entailed by any genuine successes of a new one, in the sense specified above, and one could hence reasonably maintain that older successes have no say in the estimation of the new theory's success."

Otherwise it fails to make contact with the previous paragraphs and the subsequent discussion.

- "random mean squared" in the paragraph following equation 16 needs to be "root mean square"

Publisher's Note Springer Nature remains neutral with regard to jurisdictional claims in published maps and institutional affiliations.

The original article can be found online at https://doi.org/10.1007/s11229-018-01925-9.

$凶$ Florian J. Boge

fboge@uni-wuppertal.de

1 Interdisciplinary Centre for Science and Technology Studies (IZWT), Bergische Universität Wuppertal, Gaußstr. 20, room S.11.19, 42119 Wuppertal, Germany

2 Institute for Theoretical Particle Physics and Cosmology, RWTH Aachen University, Otto-Blumenthal-Straße, 52074 Aachen, Germany 